CLINICAL IMAGES

\title{
A Rare Case of Temporal Scalp Dermoid Cyst with No Intracranial Extension
}

\author{
Hatim Belfquih, $M D^{1^{*}}$, Hassan Baallal, $M D^{1}$ and Adil Arrob, $M D^{2}$ \\ ${ }^{1}$ Department of Neurosurgery, Avicenne Military Hospital, Cady Ayyad University, Marrakech, Morroco \\ ${ }^{2}$ Department of Plastic and Maxillo-Facial Surgery, Avicenne Military Hospital of Marrakech, Cady Ayyad University, \\ Marrakech, Morroco
}

*Corresponding author: Hatim Belfquih, MD, Department of Neurosurgery, Avicenne Military Hospital, Cady Ayyad University, Marrakech, 40000, Morocco, Tel: +(212)-661-338-623

\begin{abstract}
Dermoid Cysts are considered congenital lesions, but not all of them are diagnosed at birth. Dermoid cyst involving subgaleal temporal area with no intracranial extension is a rare scalp swelling.

We report a case of 37-year-old female patient presented with a history of a scalp swelling since birth. The swelling increased in size progressively. Magnetic resonance imaging of the brain revealed extracranial cystic lesion over the temporal bone with no intracranial extension. Cyst was excised completely with no postoperative complications. On histopathology it was dermoid cyst.

Early resection mayavoid more extensive surgery, and a shorter skin incision with less risk of wound infection mayallow an improved cosmetic outcome.
\end{abstract}

\section{Keywords}

Temporal bone, Cyst, Dermoid, Scalp

\section{Introduction}

We report a case of 37-year-old female patient presented with temporal scalp swelling since birth. The swelling increased in size very slowly and progressively. On examination, there was a soft, fluid-filled lesion over the temporal bone, which was non tender and non pulsatile. There was no bruit over the swelling. Magnetic resonance imaging of the brain revealed extracranial well-defined cystic swelling over the left temporal bone with no intracranial extension, measuring approximately $20 \times 45 \times 30 \mathrm{~mm}$ in transverse, anterior-posterior, and craniocaudal dimensions. It was cystic in appearance, with hyperintense signals on T2-weighted sequence, with no flow voids (Figure 1a) and hypointense signals on T1-weighted sequences (Figure 1b) showing no surrounding edema on fluid-attenuated inversion recovery (FLAIR). There was no contrast enhancement (Figure 1c). Intraoperatively, a soft, cystic, fluid filled lesion over temporal bone, which was densely adherent to underlying tissue (Figure 1d). Cyst was excised completely with no postoperative complications. On histopathology it was dermoid cyst.

Dermoid cysts are considered congenital lesions, but not all of them are diagnosed at birth [1]. Dermoid cysts are benign soft tissue tumors that develop from abnormal sequestration and inclusion of the surface ectoderm along the lines of skin fusion during embryologic development [2]. In the literature, midline scalp cysts present a higher probability of intracranial extension because they are on a line of embryonic fusion. Early resection mayavoid more extensive surgery, and a shorter skin incision with less risk of wound infection mayallow an improved cosmetic outcome [3].

\section{Conflicts of Interest}

The author has no conflicts of interest to declare. 

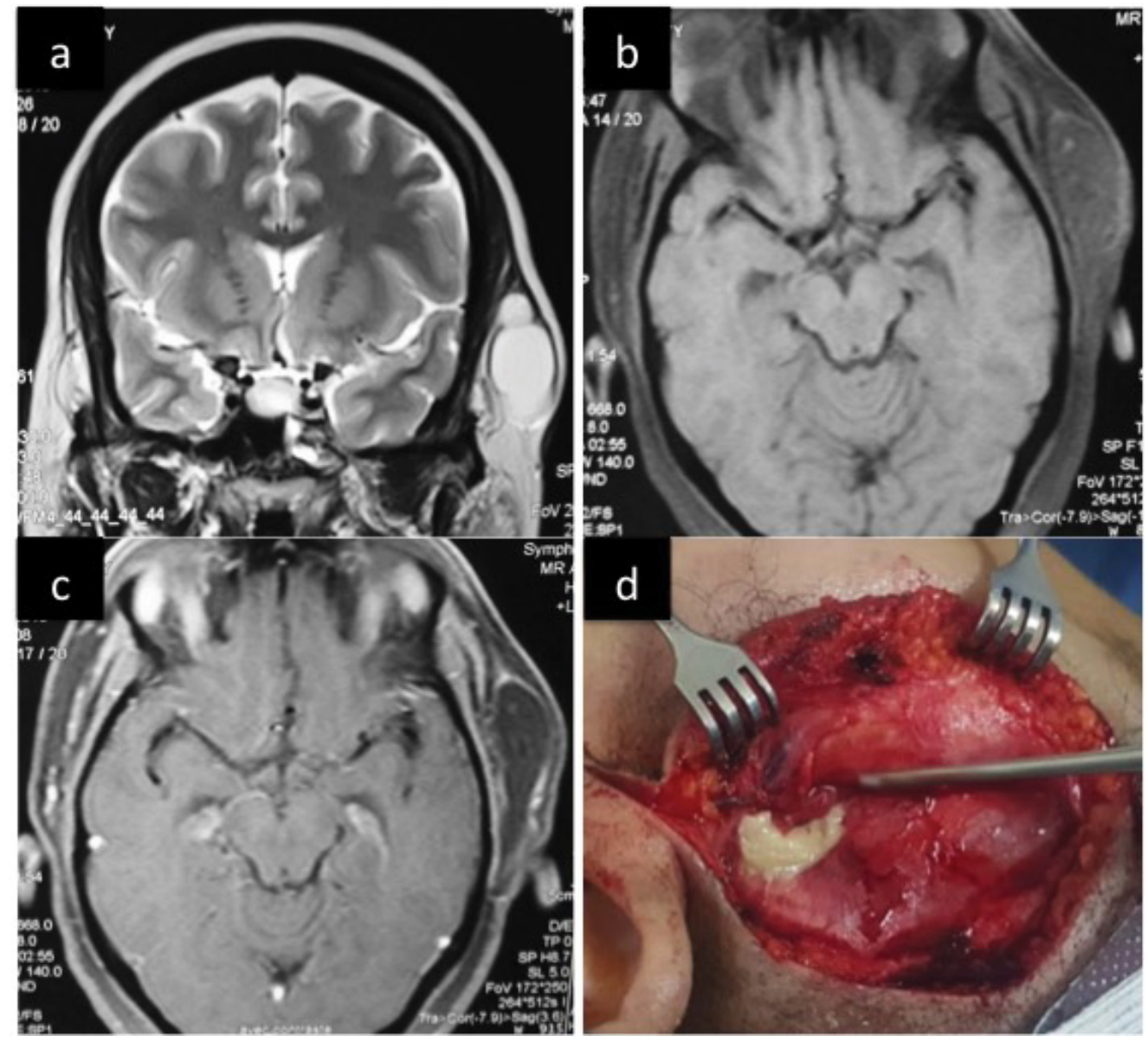

Figure 1: It was cystic in appearance, with hyperintense signals on T2-weighted sequence, with no flow voids (Figure 1a) and hypointense signals on T1-weighted sequences (Figure 1b) showing no surrounding edema on fluid-attenuated inversion recovery (FLAIR). There was no contrast enhancement (Figure 1c). Intraoperatively, a soft, cystic, fluid filled lesion over temporal bone, which was densely adherent to underlying tissue (Figure 1d).

\section{References}

1. Orozco-Covarrubias L, Lara-Carpio R, Saez-De-Ocariz M, Duran-McKinster C, Palacios-Lopez C, et al. (2013) Dermoid cysts: A report of 75 pediatric patients. Pediatr Dermatol 30: 706-711.
2. Reissis D, Pfaff MJ, Patel A, Steinbacher DM (2014) Craniofacial dermoid cysts: Histological analysis and intersite comparison. Yale J Biol Med 87: 349-357.

3. Khalid S, Ruge J (2017) Considerations in the management of congenital cranial dermoid cysts. J Neurosurg 20: 30-34.

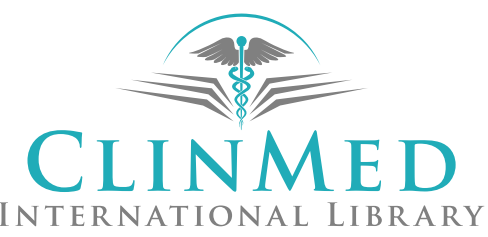

